\title{
Quantitative Determination of the Nonlinear Pinning Potential for a Magnetic Domain Wall
}

\author{
D. Bedau, M. Kläui, * M. T. Hua, S. Krzyk, and U. Rüdiger \\ Fachbereich Physik, Universität Konstanz, Universitätsstrasse 10, 78457 Konstanz, Germany
}

G. Faini and L. Vila

CNRS, Phynano team, Laboratoire de Photonique et de Nanostructures, Route de Nozay, F-91460 Marcoussis, France

\begin{abstract}
Using microwave currents, we excite resonances of geometrically confined pinned domain walls, detecting the resonance by the rectification of the microwave current. By applying magnetic fields, the resonance frequency of the domain wall oscillator can be tuned over a wide range. Increasing the power leads to a redshift due to the nonlinearity of the system. From this frequency shift, we directly deduce the quantitative shape of the potential, so that a complete characterization of the pinning potential is obtained.
\end{abstract}

Laterally confined magnetic domain walls exhibit a range of novel physical effects and are also promising candidates for applications in memory devices [1] as well as in logic circuits [2].

To use domain walls in devices, the walls have to be pinned controllably at well-defined pinning positions. Examples of artificial pinning sites include notches [3-6] and protrusions [7], both creating attractive potentials for domain walls. Domain walls can be moved between different pinning sites to implement, e.g., logic operations [2] or storage [1]. Another application was proposed by He and Zhang [8], who suggest to use a localized domain wall oscillator as a tunable microwave source. But so far it is unclear to what extent the frequency of such an oscillator can be tuned, which is one of the key requirements for applications.

For reliable operation of devices based on domain wall motion or resonance, in addition to well-defined pinning potentials, also sufficiently low critical current densities and sufficiently fast switching are required. It was shown that resonant excitations [9] as well as resonant pulse trains [10] allow for a significantly lowered threshold current density. To further understand and control domain wall dynamics, the quantitative shape of the pinning potentials has to be determined. In addition to engineered pinning centers, pinning at defects intrinsic to the material or caused by the processing is one of the key problems and obstacles for device applications, and only quantitative information about the pinning potential will lead to a further understanding of the dynamics of such random pinning processes. So an in-depth understanding of the pinning potential landscape due to constrictions but, in particular, due to intrinsic defects is a key requisite to further development in the field of domain wall dynamics.

Depending on the type of the domain wall, the dynamic behavior of pinned domain walls is determined by different contributions of the wall spin structure. While in the case of transverse walls the dynamic behavior is determined mainly by the motion of the entire wall, for vortex walls, the singularity in the center of a magnetic vortex, which points out of the plane and is one of the smallest confined magnetic structures present in domain walls, plays a key role.

A force acting on such a vortex core will cause a movement of the vortex core in the direction perpendicular to the force $[11,12]$. Therefore, if excited by a continuous ac field or current, the vortex core will carry out a gyrotropic motion around the potential minimum [13]. Depending on the regime, the vortex core will follow either a circular orbit [13] or a more complicated nonlinear orbit of higher order [14] with characteristic eigenfrequencies. In the case of a single pulse excitation, the vortex core will relax afterwards into the potential minimum on a spiral trajectory.

Such a behavior lends itself to a description of the vortex core as a quasiparticle, which oscillates in a potential defined by the geometry of the structure; i.e., the dynamic behavior of the vortex domain wall is described approximately by the motion of the vortex core. The masses of the domain wall quasiparticles have been determined in Refs. $[9,15]$, and the trajectory of the quasiparticle can be described using Thiele's equation [16]. Thiele's equation treats the spin configuration as rigid, which is acceptable in many cases, as the equations are not very sensitive to small deviations of the spin distribution. In Fig. 1(c), we present four simulated snapshots of the vortex core motion when a vortex core in a pinned vortex wall (by an artificial notch) is excited resonantly. It can be seen that the vortex core moves on an elliptical trajectory as predicted by the model [16].

Experimentally, both vortex and transverse walls have been observed in nanowires, and the domain wall types have been determined for different geometries [17]. For both domain wall types [3], the depth [4,5,7], the width [6], and the location [18] of the pinning potential have been previously determined. Using a resonant excitation of a vortex wall, the trajectory of the vortex core $[9,19]$, as well 

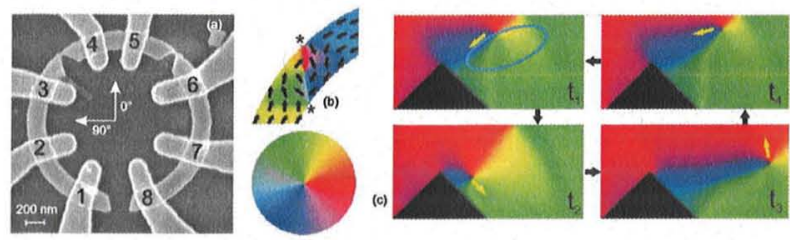

FIG. 1 (color online). (a) Scanning electron micrograph of the device with numbered contacts. Micromagnetic simulation of a vortex wall far from the constriction (b). The two stars indicate the topological edge defects, and the resonant mode corresponds to the vortex core moving on an elliptical trajectory along the line connecting the two stars. The color circle indicates the direction of the magnetization. (c) Four frames of a micromagnetic simulation of the vortex core trajectory. The arrows indicate the direction of the core motion. The duration of one full cycle is $2.5 \mathrm{~ns}$.

as the core polarity [19], has been determined, but so far the key information, namely, the quantitative determination of the shape of the pinning potential, has not been achieved.

In this Letter, we investigate the potential landscape around a pinning site by analyzing current-induced domain wall excitations in the frequency range from $100 \mathrm{MHz}$ up to $2 \mathrm{GHz}$. Using a homodyne detection scheme [9], we measure the resonance frequency of the domain walls, which corresponds to the potential well curvature. By applying external fields, we can tune the well curvature and thus the resonance frequency over a wide range from 250 to $500 \mathrm{MHz}$. In addition to using a field, we find that we can also tune the resonance frequency by varying the power, which reveals the nonlinearity of the domain wall oscillator. Using a one-dimensional anharmonic oscillator model, the power dependence of the resonance frequency is explained, and from these data we are finally able to quantitatively determine the absolute shape of the potential well around a pinning site.

For the experiments we use $25 \mathrm{~nm}$ thick and $200 \mathrm{~nm}$ wide Permalloy ring structures with $2 \mu \mathrm{m}$ outer diameter and $10-30 \mathrm{~nm}$ wide constrictions [9]. On top of the ring structure, nonmagnetic $\mathrm{Au}$ contacts are patterned, and a scanning micrograph image of the device is shown in Fig. 1(a).

Domain walls in such structures are head-to-head $180^{\circ}$ walls with a vortex or a transverse spin structure $[17,20]$, and by applying an in-plane magnetic field they can easily be generated and positioned at any desired position in the ring [6].

The domain wall is positioned along a direction of $80^{\circ}$, between contacts 2 and 3 in Fig. 1(a). From the geometry we expect a vortex wall [Fig. 1(b)] [17], as has been confirmed by micromagnetic simulations with the OOMMF code [21] and the LLG micromagnetic simulator software [22] (parameters: $K=0, M_{S}=800 \times 10^{3} \mathrm{~A} / \mathrm{m}$, $A=13 \times 10^{-12} \mathrm{~J} / \mathrm{m}, 5 \mathrm{~nm}$ cell size). The domain wall type has also been verified by magnetoresistance measure- ments using a current of $5 \mu \mathrm{A}$ injected between contacts 2 and 3 , as described in [3].

The resonance frequency is determined using the homodyne detection scheme detailed in [9]: To measure the dc component generated by the rectifying action of the domain wall, we use a lock-in detection. The microwave generator is modulated with a $3 \mathrm{kHz}$ square wave, to which the lock-in amplifier is synchronized. The microwaves are injected into contact 2 of the sample using a bias tee. The dc voltage signal is measured between the dc port of the bias tee and contact 3 . All measurements are carried out at $4 \mathrm{~K}$ in a flow cryostat with high frequency contacts.

Far from a notch, the finite propagation field of the domain wall is caused by an intrinsic pinning site, due to the edge roughness and other irregularities, such as grain boundaries. For an intrinsic pinning site, we can expect a symmetric potential, as shown in Fig. 2(a), with the potential eventually leveling off, far away from the pinning site. As discussed below, we are interested in such a symmetric pinning potential for a vortex wall, which would not be found in the case for the pinning at a notch which is intrinsically asymmetric for a vortex wall pinned on one side of the notch $[3,9,18]$. For this reason, we have chosen a strong intrinsic pinning site for our experiments, which provides the necessary symmetric pinning potential.

The pinning potential is described by global parameters, such as its maximum depth as measured from the depinning field [4,5] and its absolute width as determined in Ref. [6]. To completely characterize the potential landscape, though, we need to determine the local structure of the potential. In particular, oscillation phenomena are a sensitive probe for this, since they depend on the local shape of the potential landscape, such as the curvature, which determines the resonance frequency.

To experimentally ascertain the resonance frequency, the homodyne detection method presented in Ref. [9] is used and allows for probing domain wall resonances for variable external magnetic fields and microwave power
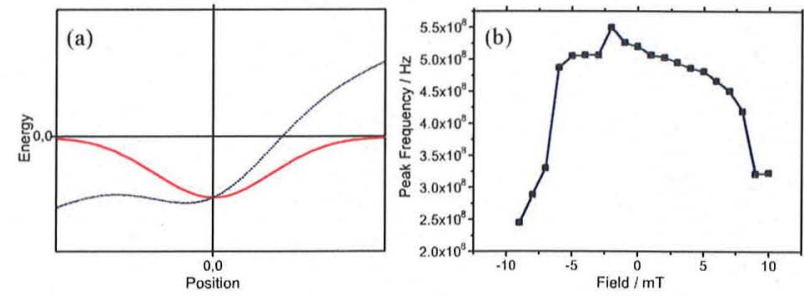

FIG. 2 (color online). (a) Schematic potential of a pinned domain wall (red solid line); 0 marks the center of the pinning potential. If an external magnetic field $B$ is applied, the potential is distorted (dotted blue line) due to the Zeeman energy contribution. This distortion could then be used to determine the undistorted potential (red solid line). (b) Measured resonance frequency of the vortex wall for different applied external fields. The curve exhibits a largely symmetric behavior, indicating a symmetric potential. For large fields, the resonance frequency decreases with increasing fields. 
levels. Furthermore, the homodyne detection scheme allows for a very precise determination of the resonance frequency compared to the depinning field method, as shown in Fig. 2(b) of Ref. [9].

By changing the injected microwave power level, we are able to change the driving force of the domain wall oscillator and therefore the amplitude of the oscillation. If the potential was perfectly harmonic, we would expect the resonance frequency to be independent of the power level applied. Frequency variations therefore provide us with a very sensitive method to directly measure the shape and any anharmonic part of the potential.

The curvature of the pinning potential can be controlled by an external magnetic field. Depending on the shape of the potential, the frequency shows a characteristic dependence on the magnetic field. For the symmetric potential shown in Fig. 2(a), the more the field is increased, the shallower the potential becomes. As the residual depth reaches a few times $k_{B} T$, we expect the domain wall to be thermally excited and to be pulled over the edge of the potential well, leading to a depinning. For a domain wall we would therefore expect the frequency to decrease for large fields, until the domain wall is depinned, at which point the dc signal disappears. The application of an external magnetic field also allows us to study the symmetry of the pinning potential, as for a symmetric potential the dependence on the field polarity will be symmetric as well.

Figure 2(b) shows the peak frequency of the rectified signal as a function of the applied external field. As we would expect, the frequency decreases with increasing applied external field. For field amplitudes larger than about $8 \mathrm{mT}$, the domain wall is depinned and no dc signal is measured, indicating that the dc signal indeed stems from the rectifying action of the domain wall. We see that, by applying a field, the domain wall oscillator can be tuned over a wide range from 250 to $550 \mathrm{MHz}$, which bodes well for using domain walls as large-range tunable oscillators.

Next we turn to the determination of the shape of the potential well, which can be determined quantitatively from the power dependence of the resonance frequency: For the potential shown as an example in Fig. 3(a), we obtain the period $T$ of the oscillation, with $U$ the potential, $m$ the quasiparticle mass, and $x$ the space coordinate, as a function of the energy $E$ stored in the system, the derivation follows the well-known textbook [23] Sec. 12:

$$
T(E)=\sqrt{2 m} \int_{0}^{E} \frac{d x_{2}}{d U} \frac{d U}{\sqrt{E-U}}+\sqrt{2 m} \int_{E}^{0} \frac{d x_{1}}{d U} \frac{d U}{\sqrt{E-U}}
$$

Integration over the energy $E$ and rearranging the terms gives us the integral equation (2). We can now directly calculate the difference between two positions $x_{1,2}$ shown in Fig. 3(a) for any given potential by the integral given in Eq. (2):
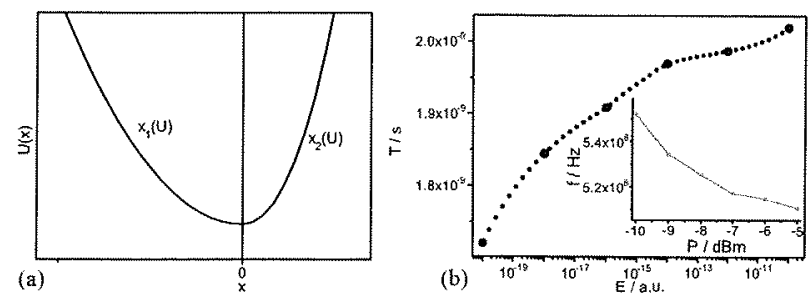

FIG. 3 (color online). (a) Schematic view of a pinning potential. For any given potential the characteristic frequencies can be computed using Eq. (1). The converse is not true; for a given $U$, only $x_{2}(U)-x_{1}(U)$ can be obtained, which means that a symmetric potential is necessary to determine the local potential curvature from the power dependence of the resonance frequency. (b) Period of the domain wall oscillator for different energies. The large blue dots are the measured values (error bars smaller than the symbols) and the small blue dots the third-order interpolation used for the numerical integration. The inset shows the measured resonance frequencies (inverse of the period) as a function of the injected power level.

$$
x_{2}(U)-x_{1}(U)=\frac{1}{\pi \sqrt{2 m}} \int_{0}^{U} d E \frac{T(E)}{\sqrt{U-E}} .
$$

If we now assume a symmetric potential, the equation simplifies to Eq. (3):

$$
x(U)=\frac{1}{2 \pi \sqrt{2 m}} \int_{0}^{U} d E \frac{T(E)}{\sqrt{U-E}} .
$$

From this equation we can directly determine the shape of any symmetric potential by numerical integration if the resonance frequency as a function of the energy is known.

Figure $3(\mathrm{~b})$ shows the oscillation period $\left(1 / f_{\text {resonance }}\right)$ as a function of the microwave power, i.e., the energy in the system. There is a clear dependence of the resonance frequency on the power, showing that the pinned domain wall exhibits a nonlinear behavior. We note that the quasiparticle model that we use works for the excitation levels that we employ, but for very high excitations the distortions of the domain wall spin structure are expected to become so large that the simple description will not suffice anymore.

Since the energy of an oscillator is proportional to the square of the driving force, the power yields the energy $E$ in the system. The driving force is due to spin torque, which is proportional to the current; therefore, the energy is proportional to the applied power. The energy dependence is shown in Fig. 3(b) (large blue disks). To determine $x(U)$ through Eq. (3), a third-order polynomial interpolation has been fitted to the data using the MATHEMATICA software packages (small blue dots).

Integrating Eq. (3) over the interpolated data shown in Fig. 3(b), we obtain the shape of the potential of the domain wall oscillator as shown in Fig. 4. The blue dots are calculated from the measured oscillator periods, and the red line indicates the parabolic part of the potential well. From the sketch of the potential in Fig. 2(a), one 


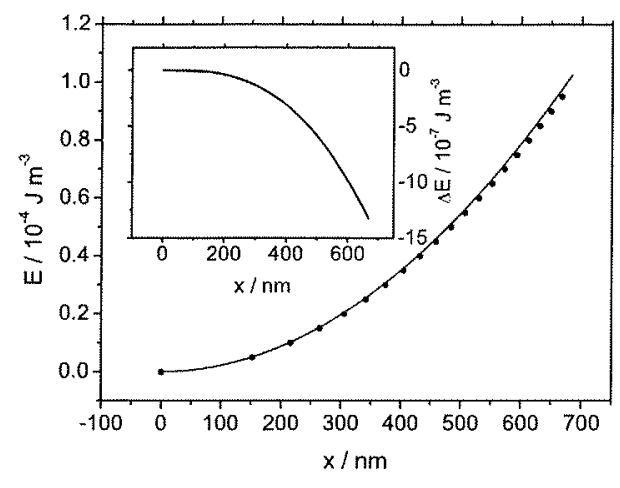

FIG. 4 (color online). Plot of the experimentally determined domain wall potential. The blue dots (error bars are smaller than the symbols) are calculated from the measured oscillation periods, and the solid red line indicates the harmonic (parabolic) part of the potential well. The inset shows the interpolated anharmonic part of the potential, which is the difference between the measured potential and the harmonic part. As expected, the domain wall potential flattens far away from the origin, indicating the anharmonic contribution to the potential.

expects that the potential flattens off far away from the minimum at the origin. And as expected, we find experimentally that the measured potential (blue dots) flattens far away from the origin, i.e., is lower than the harmonic potential (red solid line). By fitting an equation of the form $x^{2}+a x^{4}$ to the potential, we obtain a fourth-order correction of $a=8.6 \times 10^{-19}$. This anharmonic contribution to the potential has been visualized in the inset of Fig. 4 .

To absolutely calibrate the potential shown in Fig. 4, we use the methods described in Refs. [5,6] to determine the absolute depth and width of the potential. The total depth is obtained from the depinning field, $8 \mathrm{mT}$ for a vortex wall correspond to about $0.1 \times 10^{3} \mathrm{~J} / \mathrm{m}^{3}$. The total width of the potential well has been determined to be about $1400 \mathrm{~nm}$ according to the method explained in [6]. Using these values, the quantitative shape of the potential shown in Fig. 4 is ascertained, which is a complete characterization of the pinning potential.

In conclusion, we have investigated the pinning potential of a vortex-type domain wall using a homodyne detection scheme. The resonance frequency of the pinned domain wall could be tuned over a wide range by applying an external magnetic field, and the symmetry of the frequency shift with respect to the field shows that the potential is largely symmetric.

The nonlinearity of the system is reflected in the power dependence of the resonance frequency. Starting from several resonance spectra for different excitation amplitudes, the potential could be determined directly from the power dependence of the resonance frequency.

Applying an external magnetic field, the potential is modified by an additional Zeeman-like magnetostatic energy term. As the value of this Zeeman shift is known, we can completely characterize the undistorted potential for zero field from the power dependence under an external field. So as a next step this method opens up a way to completely characterize even asymmetric potentials. Since a complete characterization of any potential well is thus possible, the information can be used to completely tailor pinning potentials to a desired shape.

This work was supported by the Deutsche Forschungsgemeinschaft (SFB 513) and the Landesstiftung Baden Württemberg as well as the European Union through the Interreg IIIa program and SPINSWITCH (MRTN-CT2006-035327) and the Samsung Advanced Institute of Technology.

*mathias@klaeui.de

[1] S. S. P. Parkin, M. Hayashi, and L. Thomas, Science 320, 190 (2008)

[2] D. A. Allwood, G. Xiong, M. D. Cooke, C. C. Faulkner, D. Atkinson, N. Vernier, and R. P. Cowburn, Science 296, 2003 (2002).

[3] M. Kläui et al., Physica (Amsterdam) 343B, 343 (2004).

[4] M. Hayashi, L. Thomas, C. Rettner, R. Moriya, X. Jiang, and S. Parkin, Phys. Rev. Lett. 97, 207205 (2006).

[5] M. Kläui et al., Appl. Phys. Lett. 87, 102509 (2005).

[6] M. Kläui, C. A.F. Vaz, J. Rothman, J.A.C. Bland, W. Wernsdorfer, G. Faini, and E. Cambril, Phys. Rev Lett. 90, 097202 (2003).

[7] D. Petit, A.-V. Jausovec, D. Read, and R.P. Cowburn, J. Appl. Phys. 103, 114307 (2008).

[8] J. He and S. Zhang, Appl. Phys. Lett. 90, 142508 (2007).

[9] D. Bedau, M. Kläui, S. Krzyk, U. Rüdiger, G. Faini, and L. Vila, Phys. Rev. Lett. 99, 146601 (2007).

[10] L. Thomas, M. Hayashi, X. Jiang, R. Moriya, C. Rettner, and S. Parkin, Science 315, 1553 (2007).

[11] H. Kohno, G. Tatara, J. Shibata, and Y. Suzuki, J. Magn. Magn. Mater. 310, 2020 (2007).

[12] K. Y. Guslienko, B. A. Ivanov, V. Novosad, Y. Otani, H. Shima, and K. Fukamichi, J. Appl. Phys. 91, 8037 (2002).

[13] B. V. Waeyenberge et al., Nature (London) 444, 461 (2006).

[14] K.-S. Lee and S.-K. Kim, Appl. Phys. Lett. 91, 132511 (2007).

[15] E. Saitoh, H. Miyajima, T. Yamaoka, and G. Tatara, Nature (London) 432, 203 (2004).

[16] A. A. Thiele, Phys. Rev. Lett. 30, 230 (1973).

[17] M. Laufenberg et al., Appl. Phys. Lett. 88, 052507 (2006).

[18] D. Bedau, M. Kläui, U. Rüdiger, C.A.F. Vaz, J.A.C. Bland, G. Faini, L. Vila, and W. Wernsdorfer, J. Appl. Phys. 101, 09F509 (2007).

[19] R. Moriya, L. Thomas, M. Hayashi, Y. B. Bazaliy, C. Rettner, and S. S. P. Parkin, Nature Phys. 4, 368 (2008).

[20] R. McMichael and M. J. Donahue, IEEE Trans. Magn. 33, 4167 (1997).

[21] M. Donahue and D. Porter, OOMMF User's Guide (ITL/ NIST, Gaithersburg, MD, 2002).

[22] M.R. Scheinfein, The LLG Micromagnetic Simulator, http://llgmicro.home.mindspring.com/.

[23] L.D. Landau and E.M. Lifschitz, Mechanics (Butterworth-Heinemann, London, 1999), Vol. 1. 